\title{
The combined algorithm of venous ulcer therapy
}

\author{
Cambal $\mathrm{M}^{1}$, Labas $\mathrm{P}^{1}$, Zonca $\mathrm{P}^{1}$, Hrbaty $\mathrm{B}^{1}$ Janik $\mathrm{M}^{1}$, Polakovicova $\mathrm{S}^{2}$ \\ 1st Department of Surgery, University Hospital Bratislava, Bratislava, Slovakia. mcambal@zoznam.sk
}

\begin{abstract}
Aim: This prospective study was undertaken to find a new combined algorithm to help patients suffering from long-term chronic non-healing venous ulcers.

Methods: A total number of 20 patients suffering from chronic venous ulcers and important co-morbidities such as diabetes mellitus or cardiac failure and not responding to other therapeutic modalities were treated at our surgical department with a combination of compressive sclerotherapy, and maggot debridement therapy (MDT) using larvae of green blowfly Lucilia sericata.

Results: Using the combined therapy we have achieved a significant clinical effect in 19 patients (95\%). In 1 patient, this effect was only impermanent while 5 patients (25\%) were completely cured. Eleven patients (55\%) benefited despite incomplete healing of the ulcer. With three of our patients we have lost contact.

Conclusions: Even though the number of patients in our study is not large, we have achieved remarkable results with the combined algorithm of venous ulcer therapy. We start the healing process with compressive sclerotherapy and when this kind of therapy does not bring about the expected effect, we continue with Maggot debridement therapy. This combination of therapeutic modalities appears to be very effective and efficient (Fig. 2, Ref. 16). Full Text in PDF www.elis.sk.

Key words: ulcus cruris, compression sclerotherapy, maggot debridement therapy, MDT.
\end{abstract}

Despite the achieved progress in therapy, and availability of modern tools of "wet therapy", the deep venous insufficiency in its most complicated form - ulcus cruris (C6 - Hawai classification) still represents a serious health problem for individuals as well as for the society. At the 1st Surgical Department of the University Hospital in Bratislava, the therapeutic intervention in case of postthrombotic syndrome has a longstanding tradition based on gained experience. We recommend a therapeutic algorithm as follows.

\section{Methods}

Many patients come to us with the diagnosis of ulcus cruris. The required therapeutic intervention in case of ulcus cruris lies in biodebridement method. Most frequently, these patients have several other long-term problems related to the disease and are therefore treated by experts of other medical branches or are repeatedly hospitalised at dermatological or surgical departments or burn units but without a significant therapeutic effect. Based

${ }^{1} 1$ st Department of Surgery, University Hospital Bratislava, Bratislava, Slovakia, and ${ }^{2}$ Institute of Histology and Embryology, Bratislava, Slovakia

Address for correspondence: M. Cambal, MD, PhD, 1st Dept of Surgery, University Hospital Bratislava, Mickiewiczova 13, SK-813 69 Bratislava, Slovakia.

Phone: +421.905758448

Acknowledgement: This study came through The Promotion OP Research and Development Project for Building a Workplace Biological Therapy Design of the Technology Development and Manufacturing Biopharmaceuticals (ITMS: 26240220020), co-funded by European Regional Development Fund. on the modern pathophysiological point of view, we perceive the chronic venous insufficiency and consequential ischaemic disease of lower extremities as the cause of chronic long-term occurrence of the ulcer. Therefore complex examination of patients is essential in our approach, while the therapy includes procedures as follows:

1. Sclerotisation of insufficient perforators according to Fegan.

2. Compressive therapy based on lege artis procedures carried out for at least six weeks. adjoined by the modern „wet therapy“ of defects.

3. In case of insufficient results gained, the next step is surgical debridement.

4. Biodebridement with maggot debridement therapy (MDT) using maggots of the green blowfly - Lucilia sericata.

5. The cleansing of the defect base can be followed by plastic surgery and/or

6. Extension of modern „wet therapy“adjoined by compressive therapy.

The mentioned therapeutic procedure (Fig. 1) has proven to be very effective with our patients. Compressive sclerotherapy according to Fegan results in a reduction of venous pressure in lower extremities. For this we use sodium tetradecyl sulfate, Fibrovein, with a correctly applied compressive therapy. We have good experience with short-stretch 8 and 10 -cm wide Pütterbinde bandages with two clips applied to the leg and calf. It decreases the inflammatory infiltration and breaks the vicious circle. In many cases it results in curing the most difficult form of chronic venous insufficiency - ulcus cruris.

A correct application of compression combined with observance of treatment proceedings eliminates the subjectively per- 


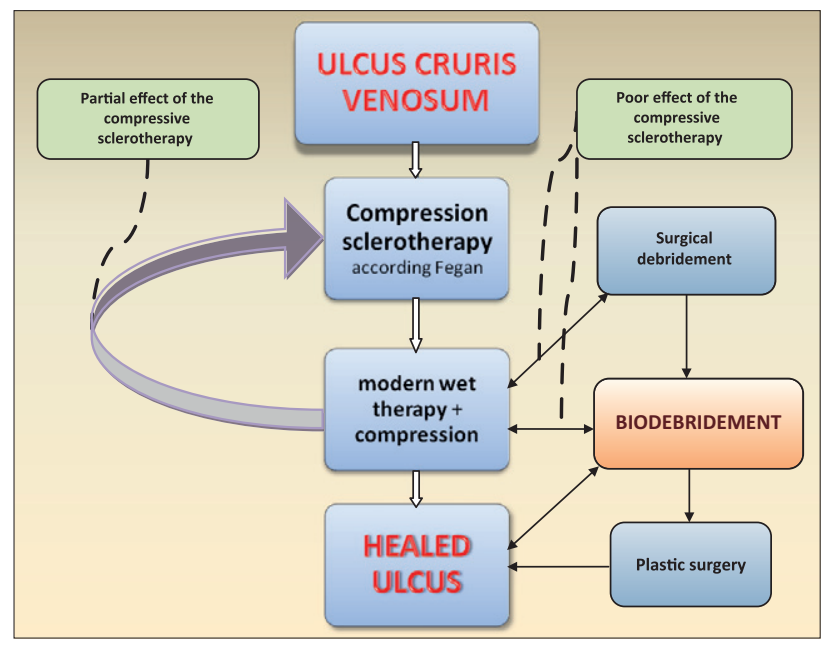

Fig. 1. Our algorithm of venous ulcer therapy.
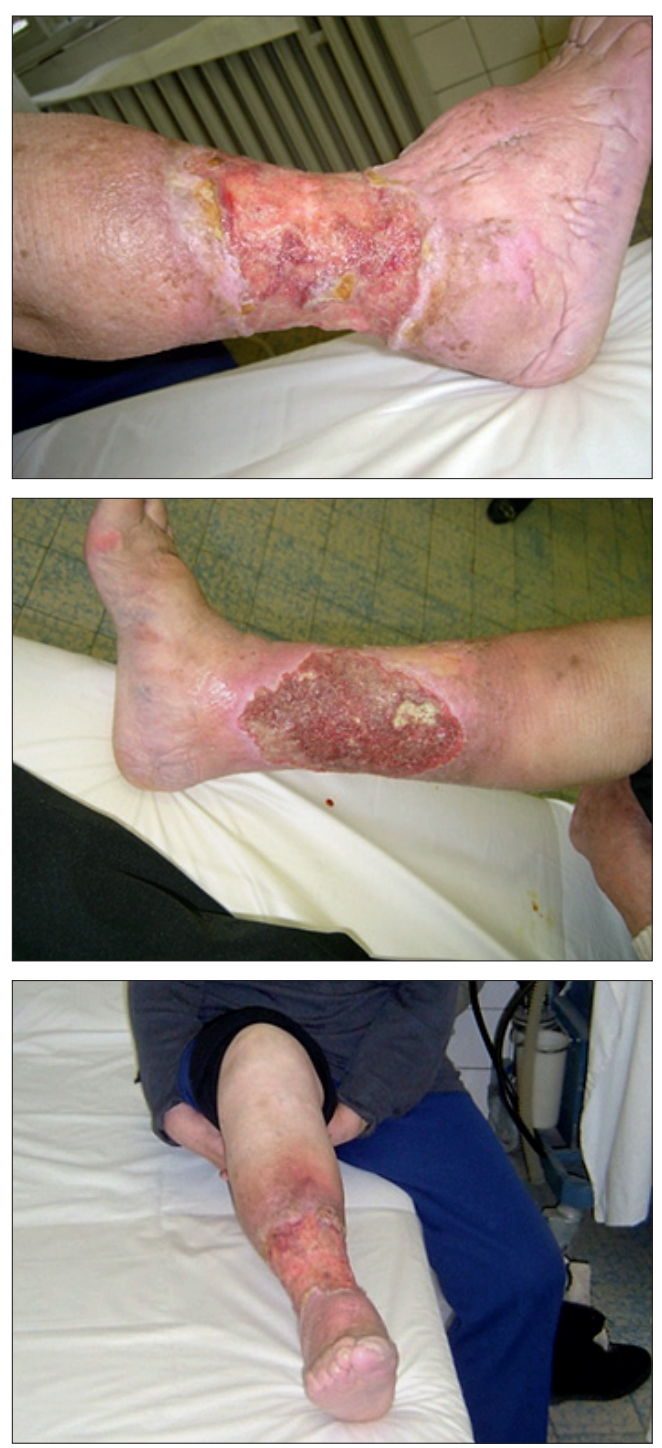

ceived difficulties of patients such as pain, swollen limbs, heaviness of lower extremities, and fluid discharge from ulcers. These improvements occur within the first days following sclerotisation of insufficient perforators. The objective finding also improves. The odour of ulcer looses its intensity, the visible presence of casing regresses, and the granulation tissue starts growing massively, followed by epithelisation occurring on the edges, and in form of islets spontaneously occurring on the ulcer.

We noticed that not every patient recovers typically as described above. Thus we are often forced to repeat the sclerotisation of insufficient perforators. An incorrectly applied compressive therapy results in re-channelling the sclerotised perforators, and resuming the mentioned vicious circle. The chronic venous insufficiency has its own course of development and progress. In many cases, the compressive therapy itself significantly reduces the pressure in the venous system of lower extremities and also alleviates some of the symptoms of chronic venous insufficiency.
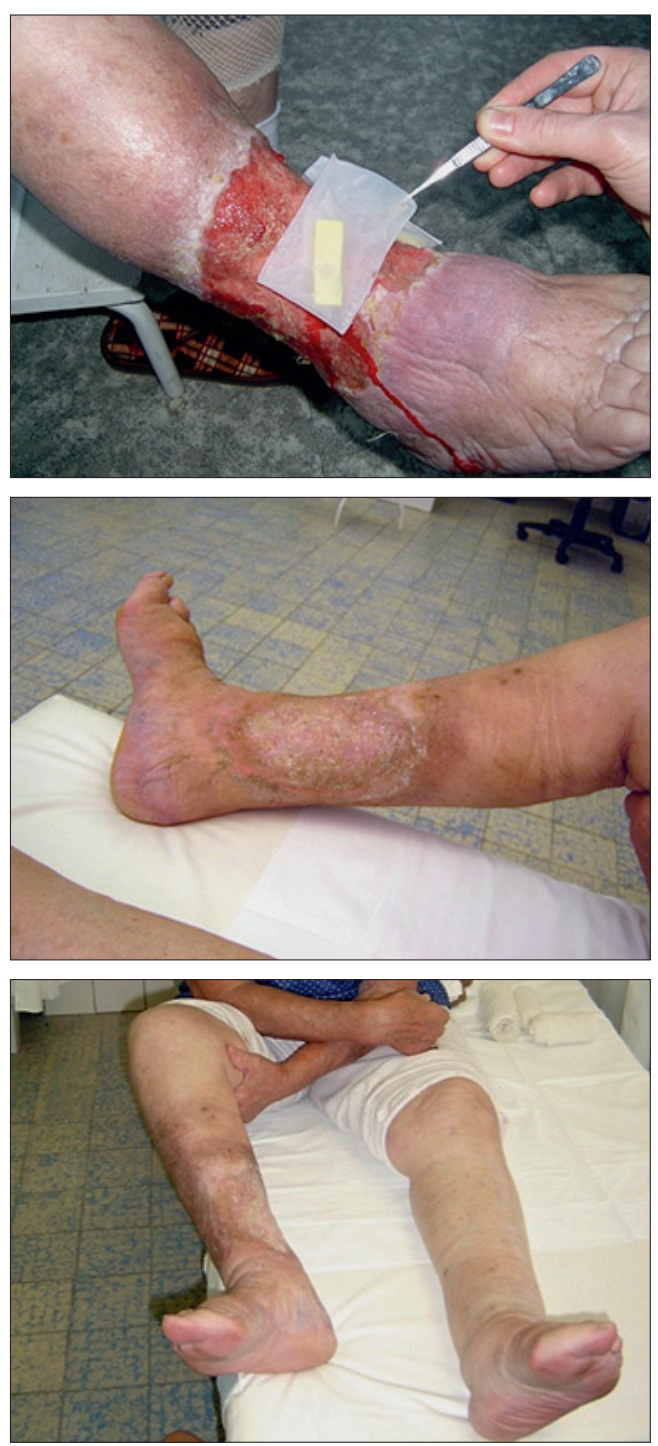

Fig. 2. Figures for example. 
The compressive sclerotherapy creates preconditions for further successful local intervention. The fact that the duration of compression should take six weeks is based on a thesis that the process of fibrotisation of sclerotised perforator takes 4-6 weeks. Should we prior to the application of larval therapy (maggot debridement therapy, MDT) observe even minimal improvements in the defect appearance, we often perform surgical debridement of solid fibrotic masses. This is done mainly in cases of large-scale fibrotic casing of the defect, as it is often colonised by numerous bacterial stems polyresistent to antibiotic therapy. Regarding the high level of pain involved in surgical debridement, we recommend it to be carried out at an operation ward (theatre) in spinal or short-term general anaesthesia of a hospitalised patient. The mentioned approach is based on the observation and experience that biodebridement is also effective in this case but it is very slow as the lytic effect of green blowfly maggots would have to dissolve an extensive layer of necrotic (fibrotic) tissue, often over $1 \mathrm{~cm}$ in thickness. The mechanical debridement which is performed before applying the biological debridement speeds up the healing process and creates the preconditions for a successful maggot therapy. Larval therapy results in eliminating the excessive necrotic/fibrotic tissue, changing the microbial population, and supporting the growth of granulation tissue.

\section{Patients}

During the past 5 years, we have treated a lot of patients with venous leg ulcers. Only a small number of them did not respond to compression sclerotherapy or responded very poorly. From this group of patients we chose 20 patients who were disposed to undergo the maggot debridement therapy. These patients were involved in a prospective study of using MDT after compression sclerotherapy (Figs for example).

Our patients' group consisted of 20 patients (10 men and 10 women), average age 63.55 years, average duration of ulcer was 13.96 years $(0.4-40)$. Thirteen patients had ischaemic heart disease, 14 patients had arterial hypertension, 3 diabetes mellitus, 6 chronic renal failure and 16 of them were obese (BMI over 30.0).

In average we used 5.2 cycles of MDT/patient in an average duration of 58.73 hours per cycle. We have used 35 MDT cycles during hospitalisation and 17 MDT cycles carried out at an outpatients clinic. Fourteen times we used direct application of MDT into the wound with modified Sherman's technique and 38 times we used "biobags".

During MDT we assessed the level of pain in scale of $0-5$, while the average value in all patients was 2.8 , which is an intermediate level of pain. In general, the pain responded very well to NSAID, however in some cases we had to use Petidine in an intramuscular dose of $100 \mathrm{mg}$.

The medicinal maggots for the whole study were provided by a non-profitable organization Medical Alternative (Medalt), the only larvae producer in Slovak Republic.

Naturally, the compressive therapy was not applied during the cycles of maggot therapy. The larvae would not survive under the compressive bandage. Immediately upon their removal, in the periods between individual cycles of MDT, the compressive therapy was applied in combination with modern methods of wet healing, as an inseparable component of the therapeutic process.

After the defect heals, we recommend a life-long compression in form of elastic bandage or compressive hose of 2nd compressive degree.

\section{Results}

In 19 patients (95\%), we have noticed a significant clinical effect of this combined therapy. In 1 patient this effect was only temporary (a very simplex severely obese hypochondriac patient, who had been suffering from venous ulcer for more than 10 years). Five patients (25\%) were completely cured and 11 patients (55\%) benefited although the healing of the ulcer was not complete. We lost contact with 3 of our patients; they did not answer our phone calls and did not respond to our mail communication.

We observed a significant wound bed cleaning, massive growth of granulation tissue after MDT, as well as epithelisation from ulcer margins and in form of epithelisation ,islets“. We have also noticed significant changes in the microbiological findings from ulcers.

After cleaning the ulcer base with MDT, two patients underwent a plastic surgery operation. The first one (suffering from a chronic circular ulcer for 30 years) had an operation according to Reverdin. Two years after this operation his ulcer was almost entirely healed. The second patient was cured two months after mesh plastic surgery.

Nineteen of 20 patients (95\%) were satisfied with their therapeutic outcome.

\section{Conclusions}

Despite the fact, that the number of patients in our study is relatively low, we have achieved remarkable results with our combined algorithm of venous ulcer therapy. In this study we present only patients, who did not benefit, or benefited only partially from compressive sclerotherapy. Then we have chosen them (with their permission) for maggot debridement therapy.

It is possible to argue that we had no control group of patients but the structure of our patients' group was so unique that it was not possible to form a control group. We definitely can compare the states of our group of patients before and after our combination of treatment modalities.

There is no doubt about the effectiveness of larval therapy. What is new in our approach is the combination of two not related methods of curing the venous leg ulcers.

We always start with the combined therapy with compressive sclerotherapy and when this kind of therapy does not bring about the expected effect, we use maggot debridement therapy. This combination of therapeutic modalities appears to be very effective and efficient in therapy of venous leg ulcers.

\section{Example}

A 72-year-old woman patient with a three-year history of a prevalent circular venous leg ulcer, repeatedly hospitalised at the 


\section{5-288}

Dermatology Unit, treated in a hyperbaric chamber without any effect. At our clinic, sclerotisation therapy was performed, after which a slight improvement of the status was visible. The patient was hospitalised due to extensive leak and scheduled for MDT with a total of five cycles of larval therapy to be applied. Two cycles were applied during hospitalisation while three therapeutic cycles in biobags were done at an outpatient department. A month after completing the MDT, the circular ulcer healed.

\section{References}

1. Bonn D. Maggot therapy: an alternative for wound infection. Lancet 2000; 356: 1174 p.

2. Brin YS, Mumcuoglu KY, Masarwe S, Wigelman RN, Gross E, Nyska M. Chronic foot ulcer management using maggot debridement and topical negative pressure therapy. J Wound Care 2007; 3: 111-113.

3. Courtenay M. The use of larval therapy in wound management in the UK. J Wound Care 1999; 8: 177-179.

4. Fleischmann W, Russ M, Moch D, Marquardt C. Biosurgerymaggots, are they really the better surgeons? Chirurg 1999; 70: 1340-1346.

5. Chan D CW, Fong D HF, Leung J YY, Patil NG, Leung G KK. Maggot debridement therapy in chronic wound care. Hong Kong Med J 2007; 5: 382-386.

6. Mumcuoglu KY, Ingber A, Gilead L, Stessman J, Friedman R, Schulman $\mathbf{H}$ et al. Maggot therapy for the treatment of intractable wounds. Int J Dermatol 1999; 38: 623-627.
7. Mumcuoglu KY. Clinical applications for maggots in wound care. Am J Derm 2001; 2: 219-227.

8. Richardson M. The benefits of larval therapy in wound care. Nurs Stand 2004; 19: 70, 72, 74 passim.

9. Sherman RA, Hall MJR, Thomas S. Medicinal maggots: an ancient remedy for some contemporary afflictions. Annu Rev Entornol 2000; 45:55-81.

10. Sherman RA, Tran JM-T, Sullivan R. Maggot therapy for venous stasis ulcers. Arch Dermatol 1996;132: 254-25

11. Sherman RA, Wyle F, Vulpe M. Maggot debridement therapy for treating pressure ulcers in spinal cord injury patients. J Spinal Cord Med 1995; 18: 71-74.

12. Sherman RA. Maggot debridement in modern medicine. Infect Med 1998; 15: 651-656.

13. Schultz GS, Sibbald RG, Falanga V, Ayello BA, Dowsett C, Harding K et al. Wound bed preparation: a systematic approach to wound management. Wound Repair Regen 2003; 11: 1-28.

14. Smith AG, Powis RA, Pritchard DI, Britland ST. Greenbottle (Lucilia sericata) larval secretions delivered from a prototype hydrogel wound dressing accelerate the closure of model wounds. Biotechnol Prog 2006; 22: $1690-1696$

15. Thomas S, Andrews A, Jones M, Church J. Maggots are useful in treating infected or necrotic wounds. BMJ 1999; 318: 807-808.

16. Wolff $\mathbf{H}$, Hansson C. Larval therapy-an effective method of ulcer debridement. Clin Exp Dermatol 2003; 28: 134-137.

Received August 20, 2010. Accepted February 14, 2012. 